\title{
Conformal Symmetries on the Horizon and Black Hole Entropy in Generic Dimensions
}

\author{
Jianwei Mei
}

\begin{abstract}
In this short note I briefly summarize some recent results regarding conformal symmetries on the horizon of general stationary and axisymmetric black holes in generic dimensions.
\end{abstract}

\section{Introduction}

In an essay in 2007, Steve Carlip used the term "universality" to reflect the fact that there exist a plethora of different ideas or calculations which (more or less) all lead to the same correct result for black hole entropy [1]. Since the discovery of the AdS/CFT correspondence it is expected that black hole entropy is related to some conformal symmetries (either at the spatial infinity or on the horizon) of the black holes [2-4]. But the term "universality" suggests a slightly more intriguing possibility, namely that one might be able to learn general lessons about the black hole entropy by studying the conformal symmetries alone, but without having to know the full detail of the underlying theory of quantum gravity. This point, if true, can be helpful in understanding the entropy of black holes in those dimensions where a consistent theory of quantum gravity (e.g. string theory) is not currently available. So it is of great importance to understand possible conformal symmetries related to black holes in detail. Ideally, one would also like to know the full detail of the dual conformal field theory (CFT) in the end.

In this short note, I briefly summarize some new results obtained during the past few years, focusing on the more detailed understanding of conformal symmetries on the horizon of general stationary and axisymmetric black holes in arbitrary dimensions.

J. Mei (凶)

Max Planck Institute for Gravitational Physics (Albert Einstein Institute),

Am Mühlenberg 1, 14476 Golm, Germany

e-mail: jwmei@aei.mpg.de 


\section{Extremal Black Holes and the Kerr/CFT Correspondence}

The new development started with the Kerr/CFT conjecture [5]. Back in 1997, Strominger already noticed that for any black hole having an $A d S(3)$ factor on its horizon, the entropy can always be calculated in the same fashion as that for the BTZ black hole [3]. In [5], the authors noticed that the near horizon limit of the extremal Kerr (NHEK) metric at a fixed polar angle is a quotient of some warped $A d S_{3}$. Since the BTZ black holes are quotients of $A d S(3)$, this close analogy indicates that what has been done for the BTZ black hole may also be done for the NHEK. Indeed, by using the techniques of Brown and Henneaux [2], the authors of [5] found that quantum gravity on the NHEK background could be dual to a chiral CFT with a central charge $c_{L}=12 \mathrm{~J}$ and a temperature $T_{L}=\frac{1}{2 \pi}$, where $J$ is the angular momentum of the Kerr black hole. Cardy's formula then reproduces exactly the Bekenstein-Hawking entropy.

Among many works that followed, one particularly important observation was made in [6]. It was noticed that the near horizon limit of all extremal black holes shares the same form of the metric, and this allows one to explain the entropy of all extremal black holes through the Kerr/CFT conjecture once and for all [7]. In a slightly different notation than [6], the common near-horizon metric is given by [7]

$$
\begin{aligned}
d s^{2}= & \frac{2 f_{r}^{0}}{\Delta_{0}^{\prime \prime}}\left[-\left(1+r^{2}\right) d t^{2}+\frac{d r^{2}}{1+r^{2}}\right]+g_{i j}^{0} d \theta^{i} d \theta^{j} \\
& +g_{a b}^{0}\left(d \phi^{a}+k^{a} r d t\right)\left(d \phi^{b}+k^{b} r d t\right),
\end{aligned}
$$

where $t$ is the time, $r$ is the radius, $\theta^{i}$ are the longitudinal angles and $\phi^{a}$ are the azimuthal angles. For the functions, $f_{r}^{0}, g_{i j}^{0}$ and $g_{a b}^{0}$ depend on $\theta^{i}$, while $\Delta_{0}^{\prime \prime}$ and $k^{a}$ are constants. The constants $k^{a}$ are related to an interesting observation [8]: for each non-vanishing angular momentum there is an independent copy of Virasoro algebra and each copy is equally good in reproducing the Bekenstein-Hawking entropy! In more detail, related to each direction $\phi^{a}$, the corresponding central charge and the temperature of the CFT are $[6,7]$

$$
c^{a}=\frac{3 k^{a}}{2 \pi} \mathscr{A}_{r e a}, \quad T^{a}=\frac{1}{2 \pi k^{a}},
$$

where $\mathscr{A}_{\text {rea }}$ is the area of the black hole horizon. Cardy formula then yields

$$
S=\frac{1}{3} \pi^{2} c^{a} T^{a}=\frac{\mathscr{A}_{\text {rea }}}{4} .
$$

Although one often prefers to understand the Kerr/CFT correspondence in relation to string theory, it also fits our theme discussed in the introduction. In particular, the near-horizon metric (1) is a blow-up of the thin region extremely close to the horizon 
of the black hole. So the conformal symmetries found in the background of the near-horizon metric must also reside on/near the horizon.

\section{Conformal Symmetries for Non-extremal Black Holes}

The Kerr/CFT correspondence, as it was originally conjectured in [5], only works for extremal black holes. This is because it is impossible to take the near horizon limit for the metrics of non-extremal black holes.

However, as Carlip has pointed out recently, there is no need to take the nearhorizon limit in order to expose the conformal symmetries [9]. Just like in [4], one can impose boundary conditions on the horizon and also recover the conformal symmetries. In [10], this was carried out for general stationary and axisymmetric black holes in generic dimensions. Similarly to the extremal case, this was made possible by identifying the common form of all the relevant metrics,

$$
d s^{2}=f\left[-\frac{\Delta}{v^{2}} d t^{2}+\frac{d r^{2}}{\Delta}\right]+g_{i j} d \theta^{i} d \theta^{j}+g_{a b}\left(d \phi^{a}-w^{a} d t\right)\left(d \phi^{b}-w^{b} d t\right),
$$

where the coordinates are the same as in (1), but the functions $f, v, g_{i j}, g_{a b}$ and $w^{a}$ now depend on both $r$ and $\theta^{i}$, while $\Delta$ only depends on $r$. More details of the metric can be found in [10]. Note that (1) is easily recovered by taking the near horizon limit of the above metric for extremal black holes. One of our main result is that, just like for extremal black holes [7, 8], in the non-extremal case there is also an independent copy of Virasoro algebra for each non-vanishing angular momentum, and each Virasoro algebra is equally good in reproducing the Bekenstein-Hawking entropy. But for non-extremal black holes, the result is off by a factor of 2 [10]. We wish to resolve this problem in the near future.

\section{Conformal Symmetries Without Boundary Conditions}

There are possible issues related to the boundary conditions used in the Kerr/CFT conjecture [11, 12]. But this does not necessarily harm the conformal symmetries, because there are other ways to expose them. For example, one can study the dynamics of a probe scalar field near the black hole horizon, and as a result people have suggested "hidden conformal symmetry" related to the Kerr black hole [13]. Further evidence of the hidden conformal symmetry was also obtained through the "subtracted geometry" [14]. In all these works the presence of the conformal symmetry is inferred from the existence of approximate $S L(2, R)$ symmetries in the radial wave equation of a probing scalar field. Inspired by these results, we then notice that for a stationary and axisymmetric black hole the on-shell Einstein-Hilbert action has a rigid $S L(m, R)$ symmetry, where $m$ is the number of ignorable coordinates ( $t$ and $\phi^{a}$ ) 
in the system. Corresponding to each rotation $\left(\phi^{a}\right)$ one has an $S L(2, R)$ subgroup from the $S L(m, R)$. We then find that each of this $\operatorname{SL}(2, R)$ is enhanced to a Virasoro algebra on the black hole horizon [15]. (See also [16] for an earlier indication.) So again one has the nice result that there is an independent copy of Virasoro algebra for each non-vanishing angular momentum.

\section{Outlook}

As said in [14] there has been little concrete progress towards a CFT interpretation of general non-extremal black holes, despite some optimistic conventional wisdom.

From the recent results of the Kerr/CFT correspondence, many fingers seem to be pointing at the existence of conformal symmetries on the black hole horizon. But much still needs to be done to expose the conformal symmetries and to explicitly show how they can account for the black hole entropy. For us, we are still off by a factor of 2 in the Carlip-like treatment, and we still have not been able to abstract any physical information from the conformal symmetries identified from the on-shell Einstein-Hilbert action on the horizon.

But if we are eventually successful with this procedure, it is likely to expose some common features in the quantum nature of black holes and such features are likely to be shared by all spacetime dimensions.

\section{References}

1. Carlip, S.: Symmetries, horizons, and black hole entropy. Gen. Relativ. Gravit. 39, 1519 (2007). doi:10.1007/s10714-007-0467-6

2. Brown, J., Henneaux, M.: Central charges in the canonical realization of asymptotic symmetries: an example from three-dimensional gravity. Commun. Math. Phys. 104, 207 (1986)

3. Strominger, A.: Black hole entropy from near-horizon microstates. J. High Energy Phys. 1998(02), 009 (1998). doi:10.1088/1126-6708/1998/02/009

4. Carlip, S.: Black hole entropy from conformal field theory in any dimension. Phys. Rev. Lett. 82, 2828 (1999). doi:10.1103/PhysRevLett.82.2828

5. Guica, M., Hartman, T., Song, W., Strominger, A.: The Kerr/CFT correspondence. Phys. Rev. D 80, 124008 (2009). doi:10.1103/PhysRevD.80.124008

6. Chow, D., Cvetič, M., Lu, H., Pope, C.: Extremal black hole/CFT correspondence in (gauged) supergravities. Phys. Rev. D 79, 084018 (2009). doi:10.1103/PhysRevD.79.084018

7. Mei, J.: The entropy for general extremal black holes. J. High Energy Phys. 2010(04), 005 (2010). doi:10.1007/JHEP04(2010)005

8. Lü, H., Mei, J., Pope, C.: Kerr-AdS/CFT correspondence in diverse dimensions. J. High Energy Phys. 2009(04), 054 (2009). doi:10.1088/1126-6708/2009/04/054

9. Carlip, S.: Extremal and nonextremal Kerr/CFT correspondences. J. High Energy Phys. 2011(04), 076 (2011). doi:10.1007/JHEP04(2011)076

10. Mei, J.: On the general Kerr/CFT correspondence in arbitrary dimensions. J. High Energy Phys. 2012(04), 113 (2012). doi:10.1007/JHEP04(2012)113

11. Amsel, A., Horowitz, G., Marolf, D., Roberts, M.: No dynamics in the extremal Kerr throat. J. High Energy Phys. 2009(09), 044 (2009). doi:10.1088/1126-6708/2009/09/044 
12. Dias, O., Reall, H., Santos, J.: Kerr-CFT and gravitational perturbations. J. High Energy Phys. 2009(08), 101 (2009). doi:10.1088/1126-6708/2009/08/101

13. Castro, A., Maloney, A., Strominger, A.: Hidden conformal symmetry of the Kerr black hole. Phys. Rev. D 82, 024008 (2010). doi:10.1103/PhysRevD.82.024008

14. Cvetic, M., Larsen, F.: Conformal symmetry for general black holes. J. High Energy Phys. 2012(02), 122 (2012). doi:10.1007/JHEP02(2012)122

15. Mei, J.: Conformal symmetries of the Einstein-Hilbert action on horizons of stationary and axisymmetric black holes. Class. Quantum Grav. 29, 095020 (2012)

16. Carlip, S.: Near horizon conformal symmetry and black hole entropy. Phys. Rev. Lett. 88, 241301 (2002). doi:10.1103/PhysRevLett.88.241301 